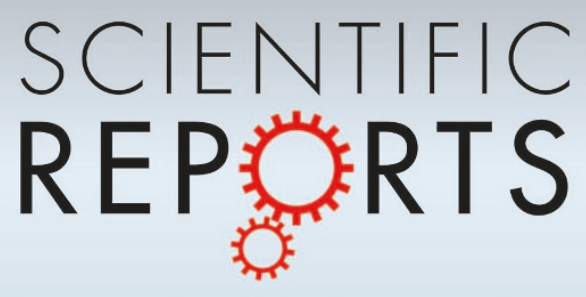

OPEN Intervention-induced enhancement in

SUBJECT AREAS:

COGNITIVE AGEING

COGNITIVE NEUROSCIENCE \title{
intrinsic brain activity in healthy older adults
}

Received

18 June 2014

Accepted

12 November 2014

Published

4 December 2014

Correspondence and requests for materials should be addressed to J.L. (lijuan@psych.ac.

$\mathrm{cn})$

* These authors contributed equally to this work.

\author{
Shufei Yin ${ }^{1,2 *}$, Xinyi Zhu ${ }^{1 *}$, Rui Li ${ }^{1,3}$, Yanan Niu' , Baoxi Wang ${ }^{1,2}$, Zhiwei Zheng ${ }^{1,2}$, Xin Huang ${ }^{1,2}$, \\ Lijuan Huo ${ }^{1,2}$ \& Juan $\mathrm{Li}^{1,3}$
}

${ }^{1}$ Center on Aging Psychology, Key Laboratory of Mental Health, Institute of Psychology, Chinese Academy of Sciences, Beijing, China, ${ }^{2}$ University of Chinese Academy of Sciences, Beijing, China, ${ }^{3}$ Magnetic Resonance Imaging Research Center, Institute of Psychology, Chinese Academy of Sciences, Beijing, China.

This study examined the effects of a multimodal intervention on spontaneous brain activity in healthy older adults. Seventeen older adults received a six-week intervention that consisted of cognitive training, Tai Chi exercise, and group counseling, while 17 older adults in a control group attended health knowledge lectures. The intervention group demonstrated enhanced memory and social support compared to the control group. The amplitude of low frequency fluctuations (ALFF) in the middle frontal gyrus, superior frontal gyrus, and anterior cerebellum lobe was enhanced for the intervention group, while the control group showed reduced ALFF in these three regions. Moreover, changes in trail-making performance and well-being could be predicted by the intervention-induced changes in ALFF. Additionally, individual differences in the baseline ALFF were correlated with intervention-related changes in behavioral performance. These findings suggest that a multimodal intervention is effective in improving cognitive functions and well-being and can induce functional changes in the aging brain. The study extended previous training studies by suggesting resting-state ALFF as a marker of intervention-induced plasticity in older adults.

$\Lambda$

ging is associated with deterioration in brain anatomy and cognitive functions, which affects older adults life expectancy and well-being ${ }^{1}$. Despite the inevitable age-related decline, the aging brain maintains plasticity, which is the capacity to modify the structure or function of the nervous system in response to environmental stimulation, impairment, or dysfunction ${ }^{2}$. Accumulating evidence has shown that older adults benefit from cognitive and physical interventions ${ }^{3,4}$, but the mechanism underlying brain plasticity is not yet clear.

A growing body of imaging studies has shown that cognitive training and exercise may alter the aging brain by inducing changes in brain structure and task-related activation. Memory training may induce changes in cortical thickness ${ }^{5}$ and activation during memory retrieval ${ }^{6}$. Specifically, memory strategy training resulted in increased activation in regions critical for strategy implementation after training ${ }^{6}$. Executive function training may induce changes in task-related activity or brain networks related to attentional control and working memory ${ }^{7}$. Exerciserelated brain alterations include structural changes in the temporal and prefrontal lobes ${ }^{8}$ and increased taskrelated activation in the prefrontal cortex ${ }^{9}$.

Recent resting-state fMRI studies have demonstrated that cognitive and physical training also changed restingstate functional connectivity ${ }^{10}$. Functional connectivity reflects the temporal coherence of intrinsic brain activity obtained in different brain regions. Intrinsic brain activity is detected as low-frequency fluctuations (LFF) in blood-oxygenation level-dependent (BOLD) signals ${ }^{11}$. Functional connectivity analysis emphasizes the interregional integration of neural networks. While most training studies have primarily focused on connectivity, it is unknown how training would affect another aspect of neural activity, regional specialization. The amplitude of LFF (ALFF) can be used as an index reflecting regional intensity of resting state activity ${ }^{12}$. Recent evidence revealed the important meaning of ALFF in both clinical and healthy populations. Abnormal ALFFs are considered as potential biomarkers used for diagnosis and treatment evaluation of neuropsychiatric disorders ${ }^{13}$, including attention deficit hyperactivity disorder, major depression, and schizophrenia ${ }^{12,14}$. In healthy adults, ALFF is associated with task-evoked brain activation ${ }^{15}$ and behavioral task performance ${ }^{16}$. ALFF has recently been used as a sensitive and reliable measure of regional intensity in aging studies.

Generally, the global strength of LFF decreases as age increases over the lifespan ${ }^{17}$. Older adults showed lower ALFF in the visual cortex during a visual task ${ }^{18}$. Negative correlations between aging and fractional ALFF (fALFF) were observed in the anterior and posterior cingulate cortex, supplementary motor area, dorsolateral prefrontal 


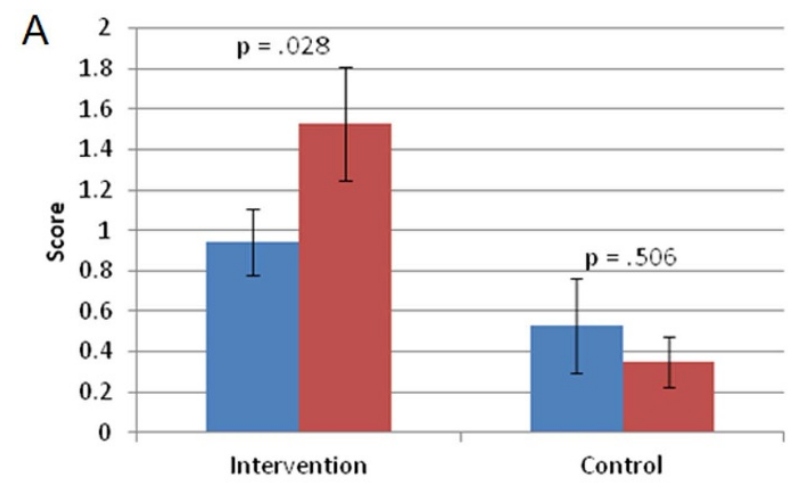

ALT difficult condition

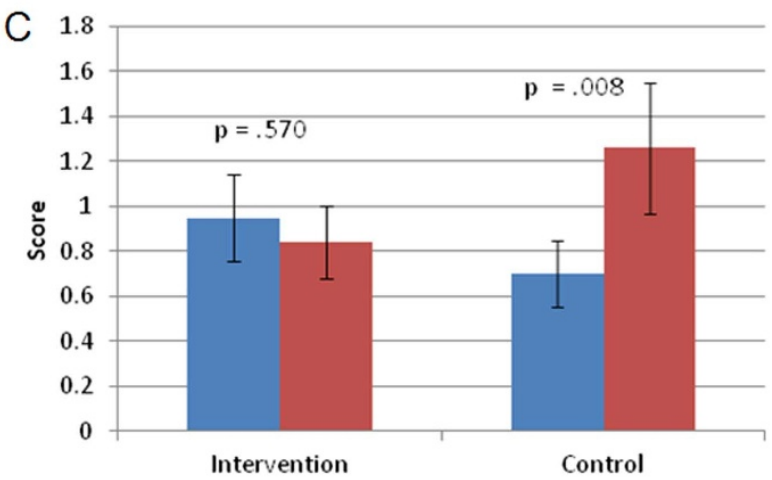

TMT

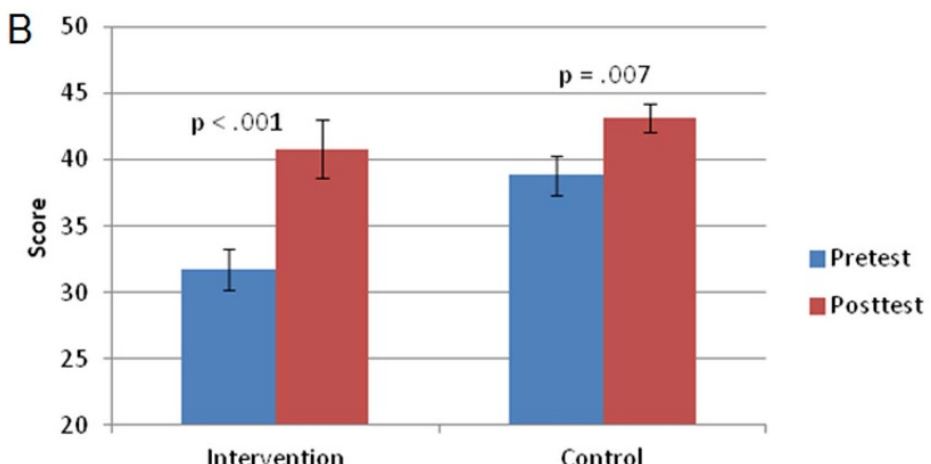

Social support

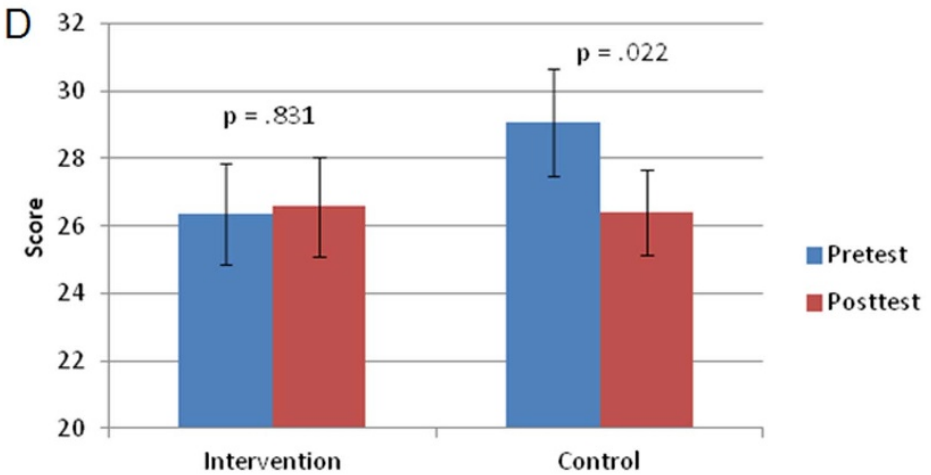

SWLS

Figure 1 Impact of multimodal intervention on behavioral performance. Bar plots show the mean scores on A) ALT difficult condition, B) social support, C) TMT, D) SWLS prior to and after intervention for the intervention and control group.

cortex, and inferior parietal lobule during cognitive tasks ${ }^{19}$. Changes in ALFF have also been associated with pathological aging, such as Alzheimer's disease (AD) and mild cognitive impairment (MCI). Older adults with MCI show reduced ALFF/fALFF in the cingulate cortex, prefrontal, and some parietal regions, and increased ALFF/ fALFF in the superior temporal gyrus ${ }^{20}$. In another study ${ }^{21}$, widespread ALFF differences was reported in the frontal, temporal, and parietal cortices among patients with $\mathrm{AD}$ and MCI compared to normal controls, and correlations between changes in ALFF and cognitive performance.

While ALFF is likely to be a significant indicator of brain function in older adults, we are not aware of any previous study using ALFF as a measure to evaluate brain plasticity. In this study, we examined the functional plasticity of ALFF in older adults after a multimodal training. Multimodal interventions are promising for older adults ${ }^{22,23}$. The multimodal approach features a combination of different modes of intervention, such as cognitive training/stimulation, physical exercise, lifestyle intervention, and stress reduction.

We designed a multimodal intervention to promote both cognitive and emotional health in healthy community-dwelling older adults. This six-week intervention combined cognitive and physical training with reminiscence group counseling, with the aim of enhancing cognitive function and well-being in the elderly individuals. We found that the multimodal intervention enhanced the functional connectivity between the prefrontal cortex and medial temporal lobe in a preceding study ${ }^{24}$. While we have found intervention-induced changes in inter-regional resting-state connectivity, how the intervention affects intra-regional spontaneous activity is still unknown. In the present study, we were especially interested in the plasticity of spontaneous brain activity, which has been indexed by ALFF in older adults. To examine the effects of multimodal intervention on spontaneous brain activity, we first performed a whole-brain voxel-wise analysis to identify the brain regions with significant intervention- related changes in resting-state ALFF. We then correlated the ALFF changes in these regions with changes in performance on behavioral tests to investigate whether the plasticity of spontaneous brain activity could predict changes in behavioral performance. Moreover, since previous studies demonstrated that pre-training individual differences had an impact on the outcome of a cognitive training task ${ }^{25,26}$, we also examined the relationship between individual differences in baseline spontaneous activity and intervention-induced changes in behavioral performance.

In summary, we examined the effects of a multimodal intervention on spontaneous brain activity in older adults using resting-state functional magnetic resonance imaging (fMRI) with the aim of evaluating the effects of this intervention in a sample of healthy older adults. We hypothesized that (1) the multimodal intervention would change the spontaneous brain activity in the intervention group; (2) intervention-induced changes in behavioral performance would be predicted by post-minus-pre changes in spontaneous activity; and (3) intervention-induced changes in behavioral performance would correlate with baseline spontaneous activity.

\section{Results}

Behavioral data of the multimodal intervention. The participants and procedures in present study have been reported in a previous study ${ }^{24}$. Analysis of the intervention effects revealed significant group $X$ intervention interactions in the ALT difficult-condition (Fig. 1A, $F_{1,31}=4.53, p=.041$ ), and social support (Fig. $1 \mathrm{~B}, F_{1,31}=5.25, p=$ .029). In addition, there was a trend toward group $X$ intervention interactions on the TMT (Fig. $1 C, F_{1,31}=3.44, p=.073$ ), and SWLS (Fig. $1 \mathrm{D}, F_{1,31}=3.46, p=.072$ ). Further analysis revealed that performance on the TMT and SWLS did not change for the intervention group $\left(F_{1,32}=.33, p=.570 ; F_{1,32}=.05, p=.831\right)$, but decreased for the control group $\left(F_{1,32}=7.95, p=.008 ; F_{1,32}=\right.$ 
$5.84, p=.022)$. Results suggested that multimodal intervention could improve or maintain cognitive ability, social support, and subjective well-being among older adults. See $\mathrm{Li}$, et al. ${ }^{24}$ for a detailed description of interventional-related performance changes.

Intervention-related ALFF changes. The whole-brain voxel-wise ANOVA identified three regions with significant group $\times$ intervention interactions (AlphaSim correction, $p<.01$ ) (Fig. 2), including the right middle frontal gyrus (MFG; peak MNI coordinate: 27, $33,-3 ; 28$ voxels), left superior frontal gyrus (SFG; peak MNI coordinate: $-12,3,66 ; 44$ voxels), and left anterior cerebellum lobe (ACL; peak MNI coordinate: $-21,-45,-33 ; 28$ voxels). Paired $t$-tests on mean ALFF in each of these three regions (ROIs) revealed that the multimodal intervention significantly increased the ALFF in right MFG $(p=.0248)$, left SFG $(p<.0001)$, and left ACL $(p=.0020)$. In contrast, the mean ALFF in all three ROIs was significantly reduced in the control group $(p<.05)$. The results suggested that there were spontaneous brain activity improvements in the intervention group compared with the control group.

Correlations between intervention-related changes in spontaneous brain activity and behavioral performance. Correlation analyses were performed to examine whether the intervention-induced changes in spontaneous brain activity were associated with changes in neuropsychological test performance (Fig. 3). We assessed relationships between brain and behavioral change using partial correlations with age and baseline behavioral performance as covariates. Intervention-induced ALFF changes in the right MFG was significantly correlated with changes in the TMT $(r=.57, p<.05$, uncorrected) and SWLS ( $r=.55, p<.05$, uncorrected). Increased ALFF in the left ACL was significantly correlated with changes in social support $(r=.55, p<.05$, uncorrected). However, after Bonferroni correction, the correlations between ALFF changes and changes in TMT, SWLS and social support were only marginally significant (ps $<.1$ ).

Individual differences in baseline spontaneous brain activity correlated with intervention-related changes in behavioral performance. We assessed relationships between baseline ALFF and behavioral change using partial correlations with age and baseline behavioral performance as covariates. The baseline ALFF in the right MFG was significantly correlated with changes in the TMT $(r=-.66, p<.05$, Bonferroni corrected) and SWLS $(r=-.70$, $p<.05$, Bonferroni corrected); predicting that individuals with lower ALFF in the right MFG would benefit more from the multimodal intervention (Fig. 4). To further confirm this result, we conducted a partial correlation analysis between the baseline ALFF and intervention-related ALFF changes in the right MFG with age as the covariate. We found that the baseline ALFF was also negatively correlated with ALFF changes in the right MFG $(r=-.69, p<.05$, Bonferroni corrected).

\section{Discussion}

In the present study, we found significant intervention effects on cognitive ability and social support. More importantly, we detected intervention-induced increases in spontaneous activation in regions of the right MFG, left SFG, and left ACL. The results demonstrated the neuroplasticity of the aging brain in response to six weeks of multimodal intervention.

Effects of multimodal intervention on cognitive function and social support. The results confirmed our hypotheses; older adults in the intervention group had significant improvements in cognitive and emotional health. Cognitive training and physical exercise have previously yielded improvements in executive function ${ }^{27}$, verbal learning, and episodic memory ${ }^{28}$ in healthy older adults. Even though aerobic exercise has strong beneficial effects on brain trophism and cognition by itself, the addition of cognitive stimuli can further enhance these beneficial effects ${ }^{29}$. Increased engagement seemed to improve the emotional well-being of participants in the intervention group. The group counseling in our intervention program emphasized the process of reviewing and interpreting positive experiences in the past and finding the sense of self and the meaning of life; that might be the main reason behind the improvement of subjective well-being ${ }^{30}$. Counseling also encouraged
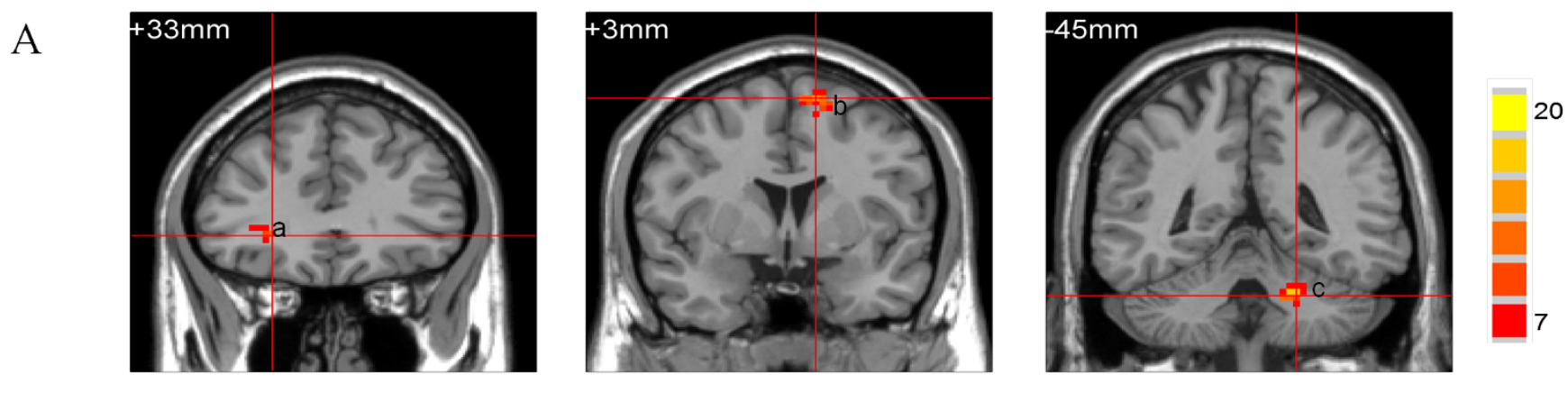

B
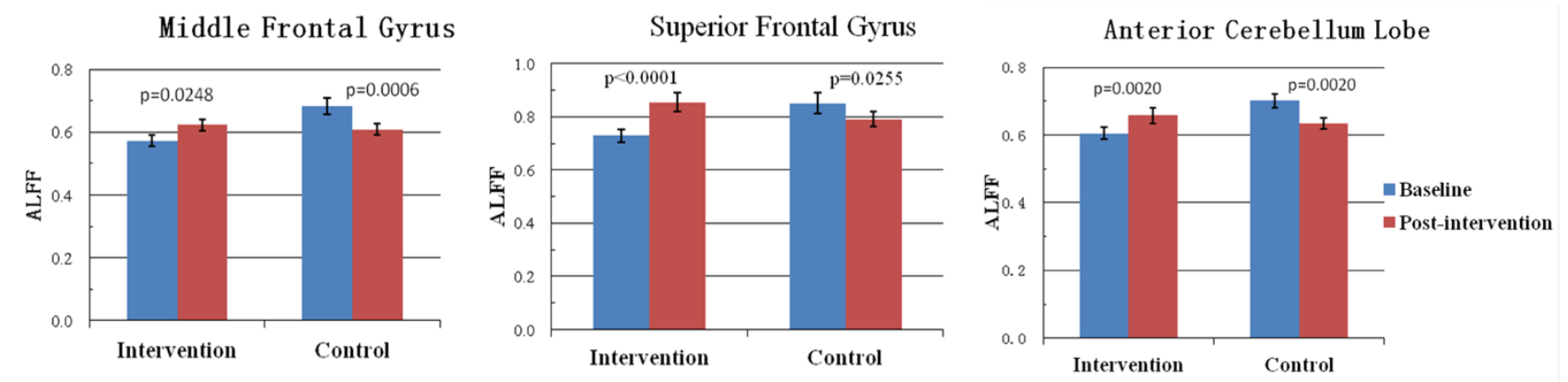

Figure $2 \mid$ Impact of multimodal intervention on MFG, SFG and ACL. (A) Coronal view of brain regions showing significant Group $\times$ Intervention interactions (AlphaSim correction, $\mathrm{p}<0.01$ ). (a) right MFG; (b), left SFG; (c), left ACL. The numbers above each image refer to the plane coordinates of MNI. Left in picture is right in the brain. (B) Bar plots show the mean ALFF prior to and after intervention for the intervention and control group. 


\section{(A) $\mathrm{MFG}$}

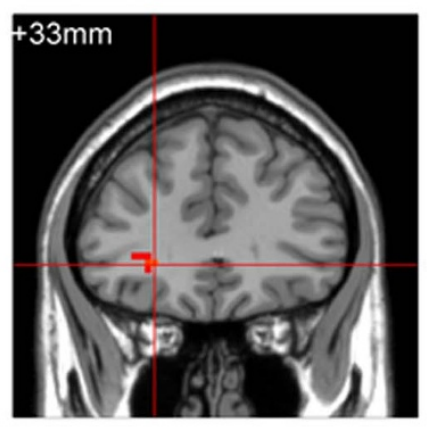

\section{(B) ACL}

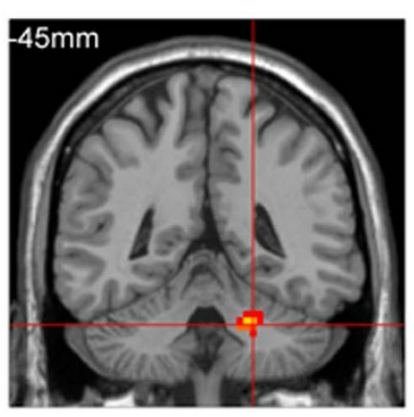

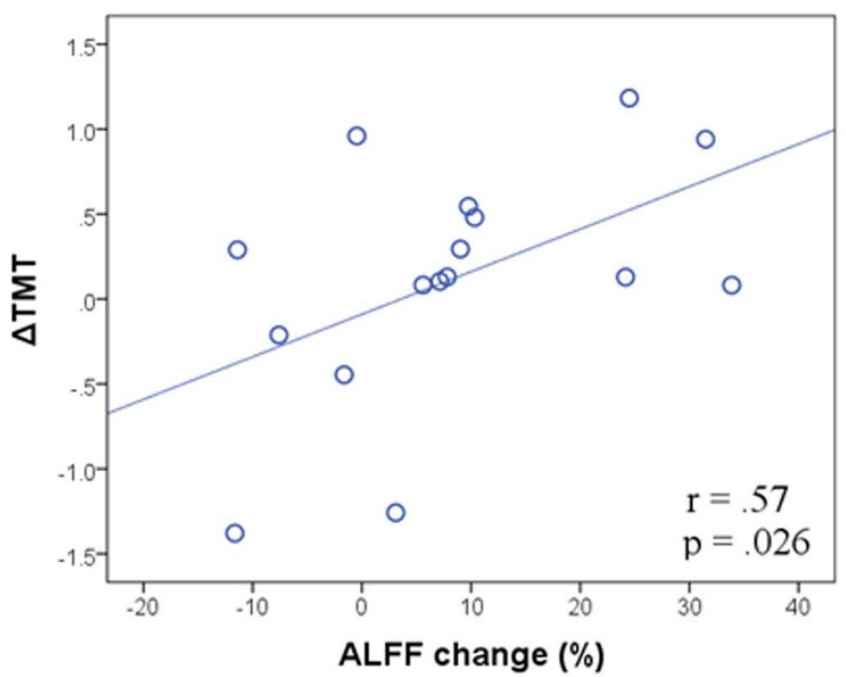
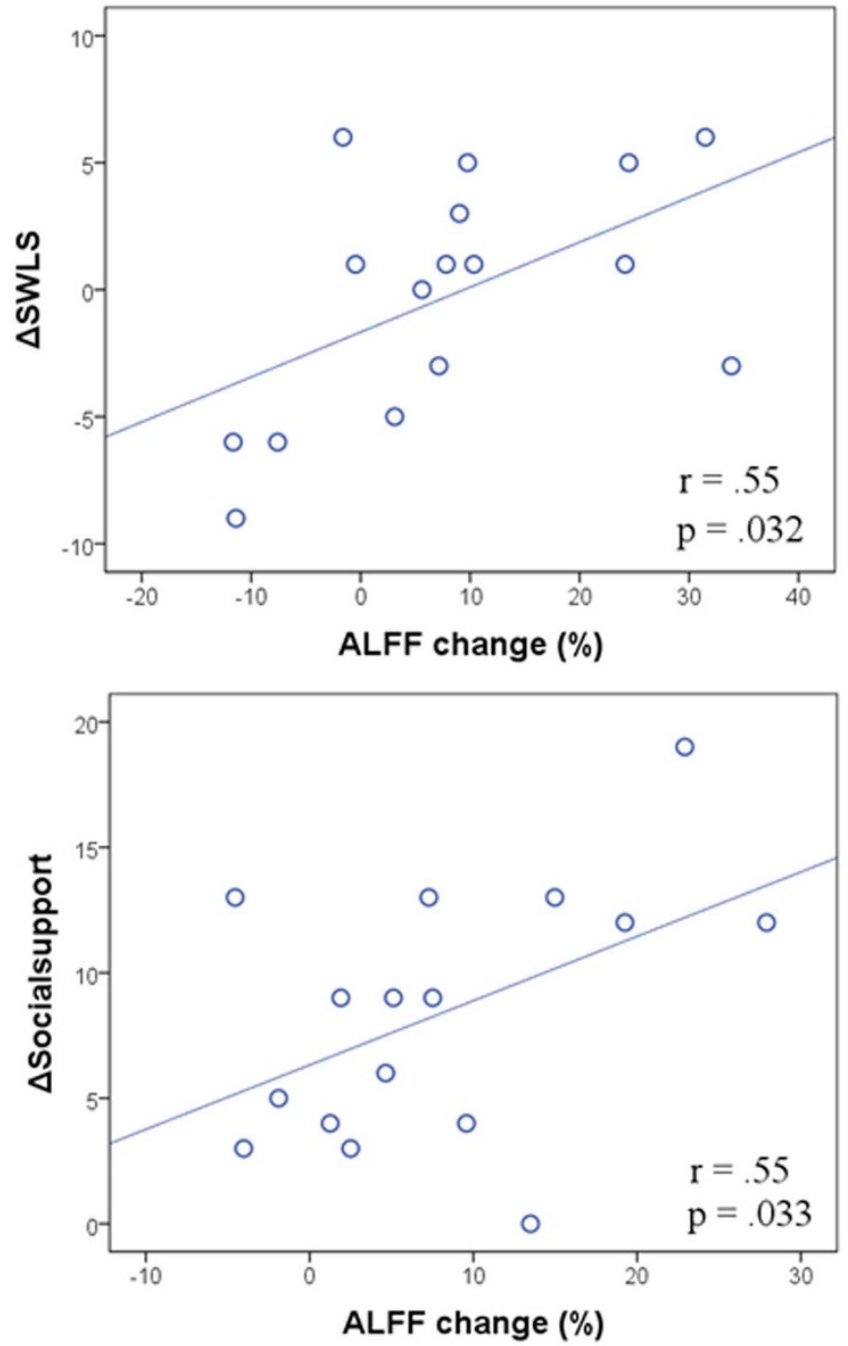

Figure 3 Spontaneous brain activity changes ([post-intervention- baseline]/baseline). (A) The right MFG was positively associated with interventionrelated changes in the TMT, the SWLS, and the social support; (B) The ACL was positively correlated with the changes in the social support.

interpersonal communication and friendship development, which might be part of the reason why the level of social support increased. In addition, the completion of the difficult cognitive and physical tasks might enhance self-esteem and inspire spiritual insight, leading to improvement in psychological well-being and social support.
Intervention induced greater spontaneous brain activity. The main finding of the current study is that the multimodal intervention resulted in greater spontaneous brain activity, which is consistent with previous studies showing that training can change resting-state brain activity or connectivity ${ }^{10}$. Research suggests that resting-state activity may assist the engagement of 

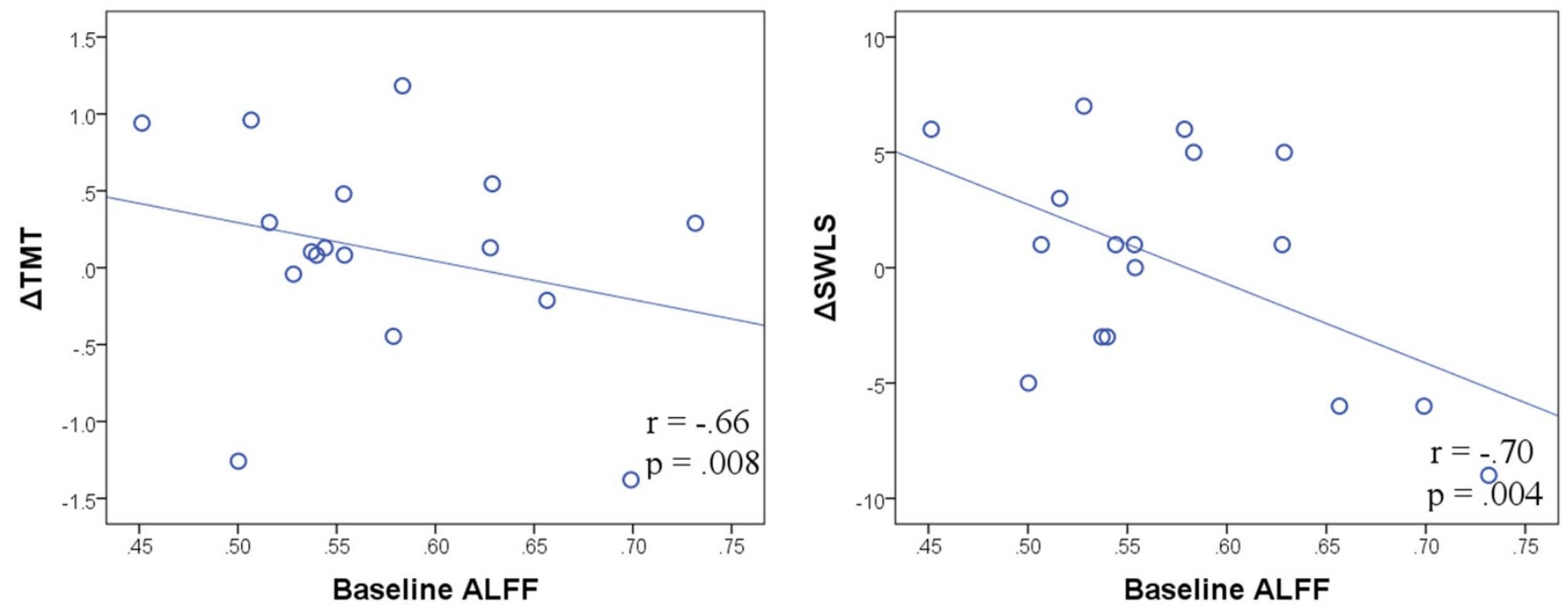

Figure $4 \mid$ The baseline spontaneous bran activity in the right MFG was negatively correlated with the intervention-related changes in the TMT and the SWLS.

brain networks involved in specific tasks ${ }^{11,28}$. There is evidence that resting-state ALFF is a predictor of task-evoked neural activity ${ }^{15}$ and behavioral performance ${ }^{16}$, and a marker of mild cognitive impairment ${ }^{20}$. In our study, older adults in the intervention group showed increased ALFF in frontal and cerebellar areas, and we believe these alterations can be interpreted as intervention-induced regional neural modifications, echoing the improvements in cognitive ability and social support in the intervention group.

The intervention and control groups showed different patterns of ALFF change in the frontal cortex and anterior cerebellum. The diminished spontaneous activity in the control group might be due to a simple test-retest effect, consistent with previous research suggesting that repeating a task without training reduces brain activation $^{31}$. Another interpretation might be that the decreased ALFF in the control group was a result of the aging process, for lifespan data have showed that ALFF for the overall brain decreases as age increases ${ }^{17}$. However, aging is a possible but much less likely explanation because six-week is rather a short period to cause significant decreases.

Intervention-induced neuroplasticity in frontal gyrus. In contrast to the control group, the intervention group showed increased spontaneous activation in regions of the right MFG and left SFG. The frontal region is one of the areas most vulnerable to aging and shows age-related structural deterioration ${ }^{31}$. Spontaneous brain activity in prefrontal regions has been negatively correlated with age $\mathrm{e}^{19}$ and was reduced in older adults with mild cognitive impairments ${ }^{20}$.

The MFG plays an important role in episodic retrieval for older adults $^{32,33}$. Specifically, the under-recruitment of the right MFG is associated with age-related declines in episodic retrieval of spatial and temporal contexts ${ }^{33}$. The method of loci, one of the mnemonics taught during the intervention, emphasizes spatial and temporal context retrieval-participants learned to memorize and recollect wordlists according to "loci," a series of sequential locations (in the present intervention, the loci were the shops and buildings on a street near the participant's residence). Increased ALFF in the MFG might reflect changes in neural activity associated with more elaborate memory retrieval in the intervention group.

The SFG is thought to contribute to higher cognitive functions, in particular to working memory and executive processing ${ }^{34}$. A restingstate fMRI study found that ALFF in the right precuneus and the medial part of left SFG was significantly correlated with individual differences in executive control, suggesting that the left SFG played an important role in executive control processing ${ }^{35}$. Working mem- ory task-evoked brain activation could be predicted by resting-state $\mathrm{ALFF}^{36}$. Resting-state ALFF in the SFG was negatively correlated with task deactivation, while resting-state ALFF in the MFG was positively associated with task activation. Together, increased ALFF in the MFG and SFG may indicate altered neural activity associated with episodic memory and working memory. This is consistent with our behavioral results that showed improved performance on associative memory and executive function tasks in the intervention group.

We conducted a correlation analysis to further examine the relationship between intervention-related changes in spontaneous brain activity and behavioral performance, and results showed that intervention-related ALFF changes in the right MFG were significantly correlated with changes in TMT and SWLS scores. The result again confirmed the effectiveness of the multimodal intervention regimen and suggested that individual differences in brain plasticity could predict the intervention-related behavioral improvements.

Intervention-induced improvements in executive function and satisfaction with life correlated with brain plasticity in the right MFG. Converging literature in cognitive neuroscience has identified the important role of the dorsolateral prefrontal cortex (DLPFC) in executive control ${ }^{37}$. The right MFG of DLPFC, for instance, has been associated with response inhibition in the go/no-go task ${ }^{38}$, as well as inhibition of imitative behavior ${ }^{39}$. Richeson et al. investigated whether individual differences in racial bias among white participants predicted the recruitment of executive attentional resources during contact with black individuals ${ }^{37}$. They found that racial bias predicted activity in the right DLPFC (including MFG) in response to black faces, and activity in this region predicted performance on Stroop test after an actual interracial interaction, and it significantly mediated the relation between racial bias and executive function performance. The results indicated that brain activity in the right MFG might also be associated with social behavior.

Satisfaction with life can be considered a positive emotion. Relative to healthy comparison subjects, depressed patients have attenuated activation in the right middle frontal gyrus, a region linked to depressive state-dependent modulation ${ }^{40}$. We speculate that our intervention led to increased activity in the right MFG, which then improved the modulation of depressive states, and increased feelings of subjective well-being. An fMRI study demonstrated that social interaction was associated with differential neural activity in the ventromedial prefrontal cortex, including the left cerebellar hemisphere and middle frontal gyrus ${ }^{41}$, which suggested an association between social behavior and frontal brain regions. In a 
similar vein, Decety and Sommerville contended that right prefrontal cortex activation could be part of a shared representation network that plays a special role in interpersonal awareness ${ }^{42}$. It is likely that this network is responsible for frontal area involvement in interpersonal awareness and communicative functions, which might then influence perceived social support. However, there is no direct evidence for this conjecture, and future studies are needed to show the existence of this possible loop.

We identified a significant negative association between baseline ALFF in the right MFG with changes in the ALFF and performance in the executive function task and SWLS. These results suggested that individuals with lower spontaneous brain activities might show greater improvement in a multimodal intervention. In addition, individual differences may play a critical role in the arrangement of an intervention regimen. Growing evidence suggests that variability in brain activation is an important indicator of brain function and cognitive performance; however, very little is known about whether ALFF is a reliable indicator of brain function and cognitive performance.

The present study suggested potential neuromarkers of intervention-induced plasticity, which need further testing. In future intervention studies, researchers should take baseline individual differences into account, including participants' brain and cognitive functions, which could help us understand how an individual would benefit from an intervention regimen. Given the importance of these individual differences, our findings support the suggestions that "one-size-fits-all" intervention regimens maybe inappropriate, and an intervention program that caters to individual differences may improve the effect of the intervention ${ }^{26}$.

A notable difference between our study and former studies is that we investigated neural plasticity from the point of functional segregation or regional specialization as indicated by ALFF. Hierarchical organization of neural activity is theorized to function in accordance with two key strategies: segregation and integration ${ }^{43}$. Recent restingstate fMRI studies are largely composed of functional connectivity analyses, which examine the interregional temporal correlation between predefined seed regions and related functional regions. Previous training studies were prone to use functional connectivity methods ${ }^{10,24,44}$, which particularly emphasized the global integration of neural networks by means of indicators reflecting interregional cofluctuations, to examine brain plasticity. The development of available analytical tools for resting-state fMRI data primarily examines integration-related connectivity measures. In our previous work ${ }^{24}$, we also used the functional connectivity method to investigate brain plasticity in older adults using the same intervention regimen as in the present study and found that individual changes in executive function and subjective well-being were significantly correlated with functional connectivity in the MFG after the intervention. Taken together with the present results, individuals with greater intervention-related improvements in TMT and SWLS achieved higher levels of functional connectivity ${ }^{24}$ and greater ALFF increases in the MFG. These consistent results indicated that ALFF, which represented functional segregation, is also a reliable indicator of brain plasticity.

However, functional segregation, the other aspect of the brain's functional integrity, has long been neglected by researchers. This methodological void is a consequence of challenges to reliably quantify intrinsic brain activity in the absence of exogenous stimuli ${ }^{13}$. LFF in resting-state fMRI encode physiologically meaningful indicators of intrinsic brain function in the absence of explicit input ${ }^{13}$, while the ALFF is seen as particularly reflecting the intensity of spontaneous neural activity at rest. ALFF has been used as a reliable and sensitive measure in the study of individuals with attention-deficit/hyperactivity disorder, schizophrenia, post-traumatic stress disorder, and major depressive disorder ${ }^{12,14,45}$. The introduction of ALFF provides a novel method to bridge gaps in our understanding of the brain's resting state by measuring the intervention-related alternation in amplitude of intra-regional brain activity at rest. Hence, ALFF ana- lysis extends and enriches the current understanding of brain plasticity, which primarily stems from connectivity analyses that examine global functional integration within the brain. In addition, the consistent results of previous functional connectivity analyses ${ }^{24}$ and present ALFF analyses further strengthen the reliability and validity of ALFF as an indicator of brain plasticity induced by intervention. In addition, these results indicated that analyses that combined these two methods might be more reliable in the assessment of the results of an intervention regimen directed at improving brain plasticity.

Intervention-induced neuroplasticity in ACL. In addition, the intervention group showed increased spontaneous activation in regions of the left ACL compared with the control group. The cerebellar lobe is linked to working memory ${ }^{46,47}$. ALFF was enhanced in the left ACL, indicating enhancement of neuronal activity at rest, which may be interpreted as recruitment of additional neural resources to compensate for the loss of cognitive function in other brain areas. However, the details of the compensation mechanism require further research. Intervention-related ALFF changes in the left ACL were significantly correlated with intervention-related increases in the level of social support. This suggests that the cerebellum might play an important role in the social behavior of healthy older adults. A study that investigated cerebellar pathology in children supports the role of the cerebellum as a modulator of mental and social functions ${ }^{48}$. However, there is little research investigating the association between cerebellum activity and social behavior in healthy older adults; therefore, strong evidence is still lacking.

Limitations and future directions. ALFF is regarded as reflecting the intensity of spontaneous neural activity at rest, and it has been used as a reliable and sensitive measure in the study of individuals with attention-deficit/hyperactivity disorder, schizophrenia, posttraumatic stress disorder, and major depressive disorder ${ }^{12,14,45}$. Our research extends the use of this method to examine brain plasticity in healthy older adults. Based on these and our previous work, we suggest that combining the functional connectivity analysis with the ALFF analysis might be more reliable in assessing the effectiveness of an intervention program in improving brain functions.

However, several limitations should be acknowledged. First, although the multimodal intervention led to benefits in cognitive functions, emotional health, and spontaneous brain activity, the mechanism underlying the effects of the intervention is far from clear. Due to the multimodal versus control comparison, we cannot clarify the contribution of each intervention component or the possible interactions between them in the present study. There have been three studies which compared the effects of multimodal intervention with those of single interventions. Fabre et al. ${ }^{49}$ compared the effects of combined aerobic and memory training, single aerobic training, and single memory training to the control group in 32 older adults. All three trained groups showed significant improvements in memory task performances, but the combined training group demonstrated greater post-minus-pre improvement on memory quotient relative to two single training groups. Oswald et al. ${ }^{50}$ compared the five-year effects of physical training, cognitive training and combined physical and cognitive training in a large sample of 375 older adults. The magnitude of long-term training effects on cognitive outcomes was greatest in the combined group and only this group showed fewer depressive symptoms over five years. Although these two studies supported the superiority of multimodal intervention, a recent study found the effects of combined training were equivalent to single cognitive training ${ }^{51}$. In this study, older adults were allocated to a computer-based cognitive training group, a mild aerobic training group, a combined cognitive and aerobic training group or a control group. Both single cognitive group and combined group had improvements in a range of cognitive tests, while single aerobic group showed no such improvement. 
Table 1 | Multimodal program for the intervention group

Cognitive training

Tai Chi Exercise

3 sessions per week

60 min per session

18

The Yang Style 24-form Tai Chi images, associating items (interactive imagery and sentence generation), and loci method.

Executive function training: three tablet games designed to train three components of executive function (inhibition, switching, and updating) developed for this study.

Instructor Trained instructors with a detailed training manual

(n)

An experienced Tai Chi instructor and two teaching assistants
Second, individual participants were not randomly assigned to the intervention or control group. Although we found no significant group difference in the baseline characteristics, we still could not rule out systematic differences between the two groups. However, if we had allocated participants from the same community to the intervention and control groups, some participants would probably know the other group's intervention contents through daily interactions, and potentially guess the study's design and purpose. Thus, to control for expectancy effect, we recruited these two groups from two similar communities, respectively, and all participants were blind to the study design. Third, although we adopted an active control, the two groups were not matched in intervention frequency and duration. The improved behavioral performance and enhanced spontaneous brain activities in the intervention group might be due in part to increased intervention frequency or duration. Although we cannot rule out this possibility, we still believe that the positive effects at least partially result from the intervention program. The intervention group had improved scores on the ALT and TMT, which are related to the memory and executive function components in the cognitive training. In view of these potential confounding factors, the design of future intervention studies should be more rigorous and include matched intervention frequency and duration for the intervention and control groups. Fourth, correlations between interventioninduced ALFF changes and behavioral changes were only marginally significant after Bonferroni correction. This lack of significance may due to small sample size. To further clarify the relationship between plasticity in spontaneous brain activity and cognition, larger samples would be needed to improve the statistical power and to verify the correlations found in the current study. Fifth, task-evoked spontaneous brain activity was not measured in the present study, so we cannot clarify how resting-state ALFF contributes to activation during task processing or compare resting-state and task-evoked brain activity, and finally, the present study did not investigate the longterm effects of intervention. Future studies should determine whether and to what extent performance gains and increases in spontaneous neuronal activity are maintained over time, which would provide evidence that the training produces consistent brain changes.

\section{Methods}

Research Design. This study was an active controlled and double blind intervention trial, and it is part of our project "Multimodal Intervention for Community-dwelling Older Adults," registered in the Chinese Clinical Trial Registry (ChiCTR-PNRC13003813). The protocol of this study was approved by the Ethics Committee of the Institute of Psychology, Chinese Academy of Sciences, and all of the participants provided written informed consent according to institutional guidelines.

Participants. The sample and procedures were the same as previous published $s^{2 t u d y^{24}}$. Forty-five healthy, community-dwelling older adults (multimodal intervention group: $\mathrm{n}=26$; control group: $\mathrm{n}=19$ ) from two communities near the Institute of Psychology, Chinese Academy of Sciences in Beijing were recruited through advertisements posted in the community service stations. After baseline evaluations, participants from one community were allocated to the multimodal intervention group and participants from the other community were treated as the control group. This allocation was made to keep participants blind to the study design.

The inclusion criteria were (1) age $\geq 60$ years; (2) education $\geq 6$ years; (3) a score $\geq 21$ on the Montreal Cognitive Assessment-Beijing Version (MoCA-BJ) ${ }^{52}$; (4) a score $<16$ on the Center for Epidemiological Survey Depression Scale (CES-D $)^{53}$; (5) right-handed; (6) free of neurological deficits or traumatic brain injury; and (7) Activities of Daily Living $(\mathrm{ADL})^{54}$ score $\leq 15$. Data from 34 participants (mean age $=$ 70.12 years, range 61-79) were analyzed, including 17 participants in the intervention group (Mean age \pm SD): $68.59 \pm 5.65$ years; 9 males) and 17 participants in the control group ( $68.59 \pm 5.65$ years; 11 males). The intervention and control groups did not differ significantly in gender, age, years of education, or on MoCA, CES-D, and ADL scores. See $\mathrm{Li}$, et al. ${ }^{24}$ for more details of participant inclusion.

Outcome measures. A broad range of tests was used to evaluate effects of training on cognitive function, social support, and subjective well-being.

Cognitive function. (1) The MoCA-BJ was used to measure global cognition. (2) Episodic memory was assessed using the Associative Learning Test (ALT) ${ }^{55}$. A list of 12 pairs of nouns was presented aurally to participants. Half of the word pairs were semantically associated (e.g., sun-moon; ALT-easy condition), and the other six were unrelated pairs (e.g., teacher-railway; ALT-difficult condition). Immediately after hearing the list, the first noun in each word pair was given as a cue, and participants were asked to recall the second noun. Participants scored 0.5 point for each correct answer in the easy condition and 1 point for each correct answer in the difficult condition. A composite ALT score that ranged from 0-9 was calculated. (3) Working memory ability was measured with the Digit Span Forward and Digit Span Backward tasks ${ }^{56}$. (4) Language ability was measured by the Category Fluency Test ${ }^{57}$. Performance on this test was measured by the total number of foods and animals named by each participant. (5) Executive function was assessed using the Trail Making Test (TMT) ${ }^{58}$. Performance on the TMT was indexed by the value of (time B time A)/time A. On the TMT, a larger score indicated a poorer performance, while greater values were associated with better performance for all other cognitive function tests.

Social support and subjective well-being. (1) The Social Support Rating Scale (SSRS) ${ }^{59}$ is a social support assessment tool widely used in China. Total SSRS scores ranged from 12-66; a higher score reflects stronger social support. (2) Subjective well-being was measured by the Satisfaction With Life Scale (SWLS) ${ }^{60}$ and the Index of WellBeing scale (IWB $)^{61}$. The SWLS is a 5 -item questionnaire that provides an overall judgment of satisfaction with one's life. The SWLS scores range from 5 (extremely dissatisfied) to 35 (highly satisfied). The IWB evaluated participants' perception of general well-being and life satisfaction. IWB scores ranged from 2.1-14.7 points.

Procedure. Participants completed the battery of assessments individually before and after the intervention. The pre- and post-scanning procedures were administered within a week after the completion of the assessments. Participants in the intervention group received a six-week multimodal intervention that included cognitive intervention, Tai Chi exercise, and group counseling. Table 1 shows the details of intervention for the intervention group. The control group attended two 120-min lectures on health and aging.

Statistical Analysis of Neuropsychological data. Baseline characteristics of the intervention and control groups were analyzed using Pearson's r, chi-square, $t$, or Mann-Whitney (nonparametric) tests. Repeated measures two-way analyses of variance (ANOVA) were conducted to examine intervention effects, with group (intervention group vs. control group) as the between-subject factor and intervention (pre- vs. post-intervention) as the within-subject factor. All statistical analyses were conducted using SPSS 19.0 (IBM Corporation, Somers, NY). 
Image Data acquisition. A 3-Tesla Siemens Trio scanner (Erlangen, Germany) equipped for echo planar imaging (EPI) at the Beijing MRI Center for Brain Research was used for image acquisition. The same fMRI protocols were used for both groups of participants within two weeks before and after intervention. During the scan, participants lay supine with their head snugly fixed by a belt and foam pads to minimize head motion, and were instructed to lie quietly, keep their eyes closed, and not to think of anything in particular. For each participant, 200 functional images were obtained using the following parameters: time repetition $(\mathrm{TR})=2000 \mathrm{~ms}$, time echo $(\mathrm{TE})=30 \mathrm{~ms}$, flip angle $=90^{\circ}$, field of view $(\mathrm{FOV})=200 \times 200 \mathrm{~mm}^{2}, 33$ axial slices, thickness $=3.0 \mathrm{~mm}$, gap $=0.6 \mathrm{~mm}$, acquisition matrix $=64 \times 64$, and inplane resolution $=3.125 \times 3.125$. In addition, a high-resolution, three-dimensional T1-weighted structural image was acquired for each participant with the following parameters: 176 slices, acquisition matrix $=256 \times 256$, voxel size $=1 \times 1 \times 1 \mathrm{~mm}^{3}$, $\mathrm{TR}=1900 \mathrm{~ms}, \mathrm{TE}=2.2 \mathrm{~ms}$, and flip angle $=9^{\circ}$.

Image processing and analysis. Resting-state fMRI data analyses were processed using the Statistical Parametric Mapping (SPM8, http://www.fil.ion.ucl.ac.uk/spm) and Data Processing Assistant for Resting-State fMRI (DPARSF) V2.0 Basic Edition $^{62}$. Preprocessing included slice timing correction, within-subject spatial realignment, between-subject spatial normalization to the Montreal Neurological Institute $(\mathrm{MNI})$ coordinate space with $3 \times 3 \times 3 \mathrm{~mm}^{3}$ re-sampling, spatial smoothing with a 4-mm Gaussian kernel, linear detrending, and temporal band-pass filtering $(0.01-0.08 \mathrm{~Hz})$. The 34 participants included in the analysis had head motions less than $2.0 \mathrm{~mm}$ in any direction, and less than $2.0^{\circ}$ of any angular motion during the resting-state $\operatorname{scan}^{12}$. Following the process of Zang et al. ${ }^{12}$, the ALFF value of each voxel was measured as the sum of amplitudes within the low-frequency range. Specifically, the time series was first converted to the frequency domain using fast Fourier transformation (FFT) to obtain the power spectrum for each voxel. Then, the square root of the power spectrum was averaged across the low frequency band ( 0.01 $0.08 \mathrm{~Hz}$ ), which was taken as the ALFF. For standardization, the ALFF value of each voxel was divided by the global mean ALFF value.

To identify brain regions that underwent intervention-related changes in spontaneous activity, the whole-brain voxel-wise group (control vs. intervention, betweensubjects) $\times$ intervention (pre vs. post, within-subjects) ANOVA (AlphaSim correction, $p<.01$ ) analysis was performed on individual ALFF maps. Age and years of education were included as confounding covariates in the statistical analyses. The regions with significant group $\times$ intervention interactions were then defined as regions of interest (ROIs). We calculated the mean ALFF of all voxels within each ROI. Paired sample $t$-tests $(p<.05)$ were then conducted on the mean ALFF to examine the effects of intervention on spontaneous activity in each ROI for the two groups. To examine the correlation of changes in intrinsic brain activity and intervention-induced behavioral improvements, we computed the partial correlations between intervention-related ALFF changes in the ROIs and intervention-related changes in behavioral performance with age and baseline behavioral performance as covariates. The significance level was set at .05 (Bonferroni corrected), but we failed to obtain any significant correlation. Since the exploratory nature of the current analysis, we further compute the correlation analysis at a relatively loose significance level $(p<.1$, Bonferroni corrected). To test whether behavioral performance changes could be predicted by baseline brain intrinsic activity, we assessed relationships between baseline ALFF and behavioral changes using partial correlations with age and baseline behavioral performance as covariates $(p<.05$, Bonferroni corrected). For all behavioral tests except TMT, intervention-related performance changes were indexed by post-minus-pre score differences. Because a larger TMT score meant poorer performance, we used pre-minus-post score difference as the index of TMT change, so that greater change scores indicated greater improvements for all behavioral tests.

1. Wilson, R. S. et al. The Influence of Cognitive Decline on Well-Being in Old Age. Psychol Aging 28, 304-313 (2013).

2. Pascual-Leone, A., Amedi, A., Fregni, F. \& Merabet, L. B. The plastic human brain cortex. Annu Rev Neurosci 28, 377-401 (2005).

3. Green, C. \& Bavelier, D. Exercising your brain: a review of human brain plasticity and training-induced learning. Psychol Aging 23, 692-701 (2008).

4. Snowden, M. et al. Effect of Exercise on Cognitive Performance in CommunityDwelling Older Adults: Review of Intervention Trials and Recommendations for Public Health Practice and Research. J Am Geriatr Soc 59, 704-716 (2011).

5. Engvig, A. et al. Effects of memory training on cortical thickness in the elderly. Neuroimage 52, 1667-1676 (2010).

6. Kirchhoff, B. A., Anderson, B. A., Smith, S. E., Barch, D. M. \& Jacoby, L. L. Cognitive training-related changes in hippocampal activity associated with recollection in older adults. NeuroImage 62, 1956-1964 (2012).

7. Anguera, J. A. et al. Video game training enhances cognitive control in older adults. Nature 501, 97-101 (2013).

8. Erickson, K. I. et al. Exercise training increases size of hippocampus and improves memory. Proc Natl Acad Sci U S A 108, 3017-3022 (2011).

9. Colcombe, S. J. et al. Cardiovascular fitness, cortical plasticity, and aging. Proc Natl Acad Sci U S A 101, 3316-3321 (2004).

10. Pieramico, V. et al. Combination Training in Aging Individuals Modifies Functional Connectivity and Cognition, and Is Potentially Affected by Dopamine-Related Genes. PLoS One 7, e43901 (2012).
11. Fox, M. D. \& Raichle, M. E. Spontaneous fluctuations in brain activity observed with functional magnetic resonance imaging. Nat Rev Neurosci 8, 700-711 (2007).

12. Zang, Y.-F. et al. Altered baseline brain activity in children with ADHD revealed by resting-state functional MRI. Brain Dev 29, 83-91 (2007).

13. Zhou, Y. et al. Spontaneous brain activity observed with functional magnetic resonance imaging as a potential biomarker in neuropsychiatric disorders. Cogn Neurodynamics 4, 275-294 (2010).

14. Liu, J. et al. Alterations in amplitude of low frequency fluctuation in treatment naïve major depressive disorder measured with resting-state fMRI. Hum Brain Mapp 35, 4979-4988 (2014).

15. Liu, X., Zhu, X.-H. \& Chen, W. Baseline BOLD correlation predicts individuals' stimulus-evoked BOLD responses. Neuroimage 54, 2278-2286 (2011).

16. Zou, Q. et al. Intrinsic resting-state activity predicts working memory brain activation and behavioral performance. Hum Brain Mapp 34, 3204-3215 (2013).

17. Zuo, X. N. et al. Lifespan trajectory of human brain functional connectome I: amplitude of low frequency fluctuations. Paper presented at $18^{\text {th }}$ Annual Meetings of the Organization for Human Brain Mapping, Beijing, China (2012, June 12).

18. Yan, L., Zhuo, Y., Wang, B. \& Wang, D. J. Loss of Coherence of Low Frequency Fluctuations of BOLD FMRI in Visual Cortex of Healthy Aged Subjects. Open Neuroimag J 5, 105-111 (2011).

19. Hu, S., Chao, H. H.-A., Zhang, S., Ide, J. S. \& Li, C.-S. R. Changes in cerebral morphometry and amplitude of low-frequency fluctuations of BOLD signals during healthy aging: correlation with inhibitory control. Brain Struct Funct 219, 983-994 (2013)

20. Han, Y. et al. Frequency-dependent changes in the amplitude of low-frequency fluctuations in amnestic mild cognitive impairment: A resting-state fMRI study. NeuroImage 55, 287-295 (2011).

21. Wang, Z. et al. Spatial patterns of intrinsic brain activity in mild cognitive impairment and alzheimer's disease: A resting-state functional MRI study. Hum Brain Mapp 32, 1720-1740 (2011).

22. Kraft, E. Cognitive function, physical activity, and aging: possible biological links and implications for multimodal interventions. Aging Neuropsychol Cogn 19, 248-263 (2012)

23. Rebok, G. W., Carlson, M. C. \& Langbaum, J. B. Training and maintaining memory abilities in healthy older adults: traditional and novel approaches. J Gerontol B Psychol Sci Soc Sci 62, 53-61 (2007).

24. Li, R. et al. Multimodal intervention in older adults improves resting-state functional connectivity between the medial prefrontal cortex and medial temporal lobe. Front Aging Neurosci 6, 39 (2014).

25. Jaeggi, S. M., Buschkuehl, M., Jonides, J. \& Shah, P. Short- and long-term benefits of cognitive training. Proc Natl Acad Sci U S A 108, 10081-10086 (2011).

26. Nikolaidis, A., Voss, M. W., Lee, H., Vo, L. T. \& Kramer, A. F. Parietal plasticity after training with a complex video game is associated with individual differences in improvements in an untrained working memory task. Front Hum Neurosci 8 , 169 (2014).

27. Persson, J. \& Reuter-Lorenz, P. A. Gaining Control Training Executive Function and Far Transfer of the Ability to Resolve Interference. Psychol Sci 19, 881-888 (2008).

28. Hötting, K., Schauenburg, G. \& Röder, B. Long-term effects of physical exercise on verbal learning and memory in middle-aged adults: Results of a one-year followup study. Brain Sci 2, 332-346 (2012).

29. Burdette, J. H. et al. Using network science to evaluate exercise-associated brain changes in older adults. Front Aging Neurosci 2, 23 (2010).

30. Pinquart, M. \& Forstmeier, S. Effects of reminiscence interventions on psychosocial outcomes: A meta-analysis. Aging Ment Health 16, 541-558 (2012).

31. Kelly, A. C. \& Garavan, H. Human functional neuroimaging of brain changes associated with practice. Cereb Cortex 15, 1089-1102 (2005).

32. Buckner, R. L., Snyder, A. Z., Sanders, A. L., Raichle, M. E. \& Morris, J. C. Functional brain imaging of young, nondemented, and demented older adults. J Cogn Neurosci 12, 24-34 (2006).

33. Rajah, M. N., Languay, R. \& Grady, C. L. Age-related changes in right middle frontal gyrus volume correlate with altered episodic retrieval activity. J Neurosci 31, 17941-17954 (2011).

34. du Boisgueheneuc, F. et al. Functions of the left superior frontal gyrus in humans: a lesion study. Brain 129, 3315-3328 (2006)

35. Xu, J. et al. Spontaneous neuronal activity predicts intersubject variations in executive control of attention. Neuroscience 263, 181-192 (2014).

36. Zou, Q. et al. Intrinsic resting-state activity predicts working memory brain activation and behavioral performance. Hum Brain Mapp 34, 3204-3215 (2013).

37. Richeson, J. A. et al. An fMRI investigation of the impact of interracial contact on executive function. Nat Neurosci 6, 1323-1328 (2003).

38. Casey, B. et al. A developmental functional MRI study of prefrontal activation during performance of a go-no-go task. J Cogn Neurosci 9, 835-847 (1997).

39. Brass, M., Zysset, S. \& von Cramon, D. Y. The inhibition of imitative response tendencies. Neuroimage 14, 1416-1423 (2001).

40. Wang, L. et al. Depressive state-and disease-related alterations in neural responses to affective and executive challenges in geriatric depression. Am J Psychiatry 165, 863-871 (2008).

41. Schilbach, L. et al. Being with virtual others: Neural correlates of social interaction. Neuropsychologia 44, 718-730 (2006).

42. Decety, J. \& Sommerville, J. A. Shared representations between self and other: a social cognitive neuroscience view. Trends Cogn Sci 7, 527-533 (2003). 
43. Zeki, S. \& Shipp, S. The functional logic of cortical connections. Nature 335, 311-317 (1988).

44. Voss, M. W. et al. Effects of training strategies implemented in a complex videogame on functional connectivity of attentional networks. Neuroimage $\mathbf{5 9}$ 138-148 (2012).

45. Yang, H. et al. Amplitude of low frequency fluctuation within visual areas revealed by resting-state functional MRI. Neuroimage 36, 144-152 (2007).

46. Stoodley, C. J., Valera, E. M. \& Schmahmann, J. D. Functional topography of the cerebellum for motor and cognitive tasks: an fMRI study. Neuroimage 59, 1560-1570 (2012).

47. Ravizza, S. M. et al. Cerebellar damage produces selective deficits in verbal working memory. Brain 129, 306-320 (2006).

48. Riva, D. \& Giorgi, C. The contribution of the cerebellum to mental and social functions in developmental age. Hum Physiol 26, 27-31 (2000).

49. Fabre, C., Chamari, K., Mucci, P., Masse-Biron, J. \& Prefaut, C. Improvement of cognitive function by mental and/or individualized aerobic training in healthy elderly subjects. Int J Sports Med 23, 415-421 (2002).

50. Oswald, W., Gunzelmann, T., Rupprecht, R. \& Hagen, B. Differential effects of single versus combined cognitive and physical training with older adults: the SimA study in a 5-year perspective. Eur J Ageing 3, 179-192 (2006).

51. Shatil, E. Does combined cognitive training and physical activity training enhance cognitive abilities more than either alone? A four-condition randomized controlled trial among healthy older adults. Front Aging Neurosci 5, 8 (2013).

52. Yu, J., Li, J. \& Huang, X. The Beijing version of the montreal cognitive assessment as a brief screening tool for mild cognitive impairment: a community-based study. BMC psychiatry 12, 156 (2012).

53. Roberts, R. E. \& Vernon, S. W. The Center for Epidemiological Studies Depression Scale: its use in a community sample. Am J Psychiatry 140, 41-46 (1983).

54. Lawton, M. P. \& Brody, E. M. Assessment of older people: self-maintaining and instrumental activities of daily living. Gerontologist 9, 179-186 (1969).

55. Xu, S. \& Wu, Z. The construction of" The Clinical Memory Test.". Acta Psychologica Sinica 18, 100-108 (1986).

56. Gong, Y. Manual of Wechsler Adult Intelligence Scale-Chinese Version. (Changsha: Chinese Map Press, 1992).

57. Strauss, E., Sherman, E. M. \& Spreen, O. A Compendium of Neuropsychological Tests: Administration, Norms, and Commentary. 499-525 (Oxford University Press, 2006)

58. Reitan, R. M. Trail Making Test: Manual for Administration and Scoring. (Reitan Neuropsychology Laboratory, 1986).

59. Xiao, S. The theoretical foundation of the social support rating scale and research applications. J Clinical Psychiatry 4, 98-100 (1994).
60. Diener, E., Emmons, R. A., Larsen, R. J. \& Griffin, S. The Satisfaction With Life Scale. J Pers Assess 49, 71-75 (1985).

61. Campbell, A., Converse, P. E. \& Rodgers, W. L. The Quality of American Life, 49-55 (New York: Russell Sage Foundation, 1976).

62. Yan, C.-G. \& Zang, Y.-F. DPARSF: a MATLAB toolbox for "pipeline" data analysis of resting-state fMRI. Front Systems Neurosci 4, 13 (2010).

\section{Acknowledgments}

This work was supported by the National Natural Science Foundation of China (31470998, 31271108, 31200847 and 31070916), Knowledge Innovation Project of the Chinese Academy of Sciences (KSCX2-EW-J-8), CAS/SAFEA International Partnership Program for Creative Research Team (Y2CX131003), and Scientific Foundation of Institute of Psychology, Chinese Academy of Sciences (Y1CX251005, 111000C038).

\section{Author contributions}

J.L. S.Y. X.Z. and R.L. together initiated the design for this paper. S.Y. and X.Z. participated in the data collection, carried out the statistical analysis, and wrote the paper. R.L. assisted with the statistical analysis of neuroimaging data. Y.N., B.W., Z.Z., X.H. and L.H. participated in the data collection. J.L. is the principal investigator of this project, and supervised the statistical analysis and the manuscript writing and revision. All authors reviewed and approved the final manuscript.

\section{Additional information}

Clinical Trial Registration: This trial was registered in the Chinese Clinical Trial Registry (ChiCTR, http://www.chictr.org) on 11/14/2013: ChiCTR-PNRC-13003813.

Competing financial interests: The authors declare no competing financial interests.

How to cite this article: Yin, S. et al. Intervention-induced enhancement in intrinsic brain activity in healthy older adults. Sci. Rep. 4, 7309; DOI:10.1038/srep07309 (2014).

This work is licensed under a Creative Commons Attribution-NonCommercialShareAlike 4.0 International License. The images or other third party material in this article are included in the article's Creative Commons license, unless indicated otherwise in the credit line; if the material is not included under the Creative Commons license, users will need to obtain permission from the license holder in order to reproduce the material. To view a copy of this license, visit http:// creativecommons.org/licenses/by-nc-sa/4.0/ 